\title{
Polyethylene/silica nanocomposites: absorption current and the interpretation of SCLC
}

\author{
K. Y. Lau' ${ }^{1,2}$, A. S. Vaughan', G. Chen' ${ }^{2}$ I. L. Hosier ${ }^{2}$, K. Y. Ching ${ }^{3,4}$ and N. Quirke \\ ${ }^{1}$ Institute of High Voltage and High Current, Faculty of Electrical Engineering, Universiti \\ Teknologi Malaysia, 81310 Johor Bahru, MALAYSIA. \\ ${ }^{2}$ The Tony Davies High Voltage Laboratory, Electronics and Computer Science, University \\ of Southampton, Southampton SO17 1BJ, UK. \\ ${ }^{3}$ Sciences, University of Reading Malaysia, 79200 Iskandar Puteri, MALAYSIA. \\ ${ }^{4}$ Engineering Sciences, University of Southampton, Southampton SO17 1BJ, UK. \\ ${ }^{5}$ Department of Chemistry, Imperial College London, London SW7 2AZ, UK.
}

\begin{abstract}
The topic of nanodielectrics continues to receive significant attention from today's dielectrics community, due to the property enhancements that can stem from the unique interfacial features within such material systems. Nevertheless, understanding of the interfacial phenomena that occur in nanodielectrics and which determine their electrical behaviour is challenging. In this paper, we report on an investigation into the absorption current behaviour of two nanocomposite systems, one containing an untreated nanosilica and the other containing the same nanofiller chemically modified using trimethoxy(propyl)silane. The results indicate that the absorption current behaviour of all the nanocomposites is very different from that of the reference, unfilled polymer; while the current flowing through the unfilled polyethylene decreased monotonically with time in a conventional manner, all nanocomposites revealed an initial decrease followed by a period in which the current increased with increasing time of electric field application. Possible mechanisms leading to the observed absorption current behaviour in the nanocomposites are discussed with the aid of space charge measurements. The presence of space charge limited conduction (SCLC) and its trap-filled limit is proposed.
\end{abstract}

Keywords: nanocomposites, nanosilica, interface, absorption current, space charge limited conduction. 


\subsection{Introduction}

The addition of a nanofiller to a polymer matrix has been shown to be a flexible means of tailoring the dielectric properties of materials [1-3] and, consequently, so-called nanodielectrics are considered to offer considerable promise as advanced material systems for use in many different application areas [4]. Indeed, thanks to the presence of the nanofiller/polymer interfaces [5-9], such material systems have been envisioned as an answer to many high voltage insulation problems [10]. For example, the partial discharge resistance, electrical treeing growth, space charge formation and dielectric breakdown performance of nanodielectrics have been compared with the respective unfilled and microfilled counterparts and worthwhile improvements in such properties have been reported [11-16].

Although two decades have passed since the nanodielectric concept was first introduced [17], many aspects of these materials are still poorly understood; Cao et al. [18] and Chen et al. [19], for example, have emphasized the unsatisfactory nature of our understanding of charge transport mechanisms. In conventional polymeric insulation, charge transport mechanisms are extremely complicated, when compared with many conducting and semiconducting materials $[20,21]$. In semicrystalline polyethylene, for example, chainfolded lamellar crystals are surrounded by amorphous conformations and there is likely to be a high concentration of traps relating to each of these structural motifs. On the addition of a nanofiller, charge transport mechanisms will become yet more complicated, since the inclusion of nanoparticles will introduce extensive interfacial surfaces between the nanofiller and the polymer. The presence of such interfaces may directly introduce additional nanofiller/polymer trapping sites and/or modify the surrounding polymer morphology, thereby affecting the local density of states within the system.

The current flow caused by the action of an applied electric field on charge carriers within a dielectric material can broadly be categorized into three types [22]: the initial current that flows through the material is the capacitive charging current, which causes a dramatic rise at the very beginning of the voltage application. This is followed by a gradual decrease of current, known as the absorption current or the anomalous current. Conventionally, the absorption current decreases slowly until it reaches a quasi-steady state, providing a conduction current that is often used to compare the conductivity of different dielectric materials. Of these, the absorption current is considered to be an important characteristic of polymers with regard to their time-domain response to a direct current (DC) poling field. This is because the results of absorption current measurements can be related to space charge 
measurements to gain a better understanding of the relationship between space charge accumulation and movement, as well as the resulting effects in the external circuit [23, 24]. Therefore, investigations into the absorption current behaviour could contribute to a better understanding of nanofiller/polymer interactions in nanocomposites, in particular, in relation to charge transport mechanisms. According to Das Gupta and Joyner [25], factors affecting absorption current include electrode polarization, dipole orientation, charge accumulation and trapping, tunnelling of charge carriers from electrodes to empty traps and hopping of charge carriers through localized states.

The current-time characteristic of the absorption current is often found to follow a power law relationship [24, 26] or, more specifically, the Curie-von Schweidler Law:

$$
I=A t^{-b_{n}}
$$

where $I$ is the current, $t$ is the time after the application or removal of the external field, $A$ is a temperature dependent factor and $b_{n}$ is a constant representing the slope of the log-log current-time plot. It should be noted that interpretation of the exponent $b_{n}$ is complicated by the fact that polymers exhibit a distribution of relaxation times which, in polyethylene for example, cannot easily be determined at normal temperatures [25].

Analysis of the exponent $b_{n}$ is, nevertheless, not uncommon and has been undertaken by some researchers in an attempt to understand those factors that affect the absorption current. For example, Mazur [27] plotted $\log I$ vs $\log t$ and found that the absorption current behaviour could be expressed by two straight lines with a break point; the break point time, $t_{p}$, and the exponent $b_{n}$ were found to depend on the polarization field. Roy [28] compared $b_{n}$ values obtained from various nanocomposites and found that those containing surface treated nanofillers exhibited lower values of $b_{n}$ compared with analogues in which the nanofiller had not been functionalised. Roy [28] also reported that unfilled crosslinked polyethylene (XLPE) and microfilled XLPE exhibited a characteristic reduction in slope that was not found in the nanocomposites. Meanwhile, Smith [24] reported that nanofilled XLPE exhibited lower values of $b_{n}$ compared with unfilled XLPE and microfilled XLPE and suggested a lower charge carrier mobility in the nanocomposites.

According to the work of Many and Rakavi [29], who studied the effects of space charge limited current in solids in the presence of trapping, the charge carrier mobility $\mu$, can be estimated using the following formula: 


$$
\mu=\frac{0.787 d^{2}}{t_{p} V}
$$

where $d$ is the sample thickness, $t_{p}$ is the time at which the change of slope in log $I$ vs $\log t$ occurs (i.e. when a charge front arrives at the electrode) and $V$ is the applied voltage. However, in generating the above equation, several assumptions have been made; for example, the effect of the diffusion current is omitted and it is assumed that the sample contains only a single trap depth. Although these assumptions will not apply to all materials, the equation has nevertheless been commonly used to estimate charge carrier mobility $[28,30$, $31]$.

In general, the current density in a dielectric film of thickness $d$ will be made up of three components and can therefore be written [32]:

$$
J=n e \mu E-e D_{n} \frac{d n}{d x}+\varepsilon_{0} \varepsilon_{r} \frac{d E}{d t}
$$

where $J$ is the current density, $n$ is the concentration of charge carriers, $D_{n}$ is the diffusion coefficient of the charge carriers, $E$ is the electric field, $e$ is the magnitude of the electronic charge, $\varepsilon_{r}$ is the relative permittivity of the material and $\varepsilon_{0}$ is the permittivity of free space. In Equation (3), the first term corresponds to charge carrier drift, the second relates to diffusion and the third displacement. Assuming, initially, that there are no thermally generated carriers, no traps, Ohmic contacts and that the current density is dominated by electron transport then, under steady state conditions with a uniform space charge distribution, substituting from Poisson's equation, rearranging and integrating gives:

$$
E=\left[\frac{2 J}{\varepsilon_{0} \varepsilon_{r} \mu}\left(x+x_{0}\right)\right]^{1 / 2}
$$

where $x_{0}$ is a constant of integration that can be estimated by considering the boundary conditions at the electrode at $x=0$. Integrating Equation (4) across the thickness of the sample and applying the usual assumption that $d » x_{0}$, finally leads to: 


$$
J=\frac{9 \varepsilon_{0} \varepsilon_{r} \mu V^{2}}{8 d^{3}}
$$

Equation (5) represents the current density associated with space charge limited conduction (SCLC) [33-36]. Including an additional Ohmic conduction term then leads to the following relationship:

$$
J=\left(n e \mu \frac{V}{d}\right)+\left(\frac{9}{8} \varepsilon_{0} \varepsilon_{r} \mu \frac{V^{2}}{d^{3}}\right)
$$

where the first part of the equation represents Ohmic conduction and the second represents space charge limited conduction (also known as the Mott and Gurney square law [37]). In Equation (6), which applies in the absence of any traps, the mobility has its conventional meaning. However, when traps are present, these will influence charge transport dynamics since the number of charge carriers available for conduction will be reduced by the factor $\theta$, which corresponds to the ratio of free charge carriers to trapped charge carriers [38]. Although this may be thought of as being "trap limited carrier concentration", it is traditionally referred to as "trap limited mobility" [32]; these two viewpoints are, however, equivalent since for Ohmic conduction, for example, the conductivity $\sigma$, would be written:

$$
\sigma=\theta n e \mu
$$

In the case of charge injection into the dielectric followed by trapping, all the injected charges will contribute to the space charge but only a fraction, $\theta$, will be available for conduction. Thus, to account for trap filling processes, Equation 6 is rewritten as follows:

$$
J=\theta\left[\left(n_{o} e \mu \frac{V}{d}\right)+\left(\frac{9}{8} \varepsilon_{0} \varepsilon_{r} \mu \frac{V^{2}}{d^{3}}\right)\right]
$$

This connection between mobility and charge trapping is well illustrated in the work of Wang et al. [39], who considered the effect of deep traps on electrical conduction and breakdown in nanodielectrics and wrote: 


$$
\mu \propto \frac{1}{1+\exp \left[\frac{\left(E_{t}-\Delta E_{F}\right)}{k T}\right]}
$$

where $E_{t}$ represents the energy of the deep traps, $\Delta E_{F}$ results from the Poole-Frenkel effect, $k$ is the Boltzmann constant and $T$ is the absolute temperature. In this approach, it was assumed that electrons could escape from shallow traps but that electrons in deep traps were unlikely either to escape from these states or to be able to tunnel from one deep trap to another.

To illustrate the above effects, a schematic log-log plot of current density as a function of applied voltage is presented in Figure 1. At low electric fields, the voltagecurrent relation tends to follow Ohm's law. In this case, two conditions are possible, namely, trap-free and trap-limited Ohmic conduction; but the latter is thought to predict more reasonable values of charge carrier mobility for real insulators, due the inevitable presence of trapping sites [32]. Above the transition voltage, $V_{t r l}$, the current becomes proportional to the square of the voltage, due to the presence of space charge limited current, thus obeying the Mott and Gurney square law. Since traps are being filled in this case (trap-filled space charge limited conduction) $\theta \neq 1$; rather, $\theta<<1$. When all the traps are filled, the trap-filled limit, $V_{t f l}$, is reached and $\theta$ changes from its small value to unity and the current density rises rapidly to approach the current density of a material without traps. Upon a further increase in voltage, the Mott and Gurney square law will resume, but the mechanism will become trapfree space charge limited conduction. The trap-filled and trap-free space charge conduction phenomena may be repeated if the material has several discrete trap levels or, in the case of a wide distribution of trapping energies, the trap-filled limit will not be well defined [32].

The above discussion briefly summarises how trapping sites and space charge affect conduction mechanisms in a dielectric, but does not consider the origin of such trapping sites. In polymers, traps are commonly considered in terms of conformational disorder and chemical defects/impurities. Wang et al. [40] used computer simulation techniques to evaluate excess electron states in various structural motifs of polyethylene and reported, at room temperature, a rapidly rising density of states with a mobility edge separating localized from delocalized states. As additional disorder was introduced into the structure, the electronic ground state was found to drop from $+0.18 \mathrm{eV}$ to $-0.37 \mathrm{eV}$ and localized electron states in the amorphous phase were associated with sub-nanometre cavities in the system. A range of chemical impurities have also been studied using similar techniques [41], which demonstrates the existence of states with trap energies ranging from 0.04 to $1.53 \mathrm{eV}$; moieties 
containing carbonyl groups or conjugated double bonds constitute the deeper traps, while those containing hydroxyl and non-conjugated double bonds equate to shallower traps. This work also considered the residence times in traps with different energies, concluding that electrons may reside in chemical trap for much greater times, by several orders of magnitude, than in physical traps.

In the case of nanocomposites, the structural and chemical complexity of the base polymer will be compounded by the nanoparticles, which will locally perturb the structure of the polymer (conformational disorder) and introduce different chemistries. Shi and Ramprasad [42] have reported modelling studies of the density of states at the interface between a vinyl silane treated silica and poly (vinylidene fluoride). This work revealed the existence of localized states at the interface that the authors associated with double bond in the vinylsilane and classified these as shallow traps for electrons and holes, due to their proximity to the conduction band and valence band edges. Evidently, the electrical response of nanoparticle interfaces is subtly dependent on such factors, which are deliberately introduced, and on the diffusion of mobile species to interfaces [43] and, consequently, the detailed study of the local structure, chemistry and electrical characteristics of such regions is in its infancy. However, since the concept of dielectric nanocomposites was first introduced by Lewis in 1994 [17], the essential characteristics of this class of material have been associated with variations in the "intensity" $I$ [5] of properties associated with local interfacial features, such that the extent of the interface/interphase is then defined by the local spatial variation in $I$. Although more explicit ideas have been proposed based upon the formation of structurally distinct interphase zones [6], these should be considered as working hypotheses. Nevertheless, the influence of such factors on charge transport dynamics in nanodielectrics has been discussed by Min et al. [44], who suggested the following: the presence of nanoparticles affects trapping energies and thereby the carrier mobility in the bulk; the conductivity of the interaction zone differs from that of the unaffected polymer; in nanodielectrics, the existence of trapping centres captures injected charges near the electrodes, thereby, affecting subsequent charge injection processes; the proposed interaction zones affects the electrode contact potential barrier.

The study reportd here set out to examine the effect of adding a commercial nanosilica filler on the absorption current behaviour of polyethylene. In our previous papers, space charge accumulation in polyethylene/silica nanocomposites has been described over a relatively short time period [45], which has demonstrated that the added nanosilica affects charge trapping; a preliminary assessment of their absorption current behaviour has also been 
presented [46]. Here, we discuss in detail the absorption current behaviour of the nanocomposites and correlate the results with space charge measurements made over much longer timer scales, in an attempt to interpret the data in the context of SCLC effects.

\subsection{Experimental}

\subsection{Materials}

The base polymer used in this study was a polyethylene blend composed of $80 \mathrm{wt} \%$ of the low density polyethylene LD100BW (ExxonMobil Chemicals) and $20 \mathrm{wt} \%$ of the high density polyethylene Rigidex HD5813EA (BP Chemicals). The nanofiller used was silicon dioxide nanopowder (nanosilica) obtained from Sigma-Aldrich, with a quoted particle size range of $10 \mathrm{~nm}$ to $20 \mathrm{~nm}$. When this nanosilica has been used as-supplied it is referred to as untreated throughout the text.

The surface chemistry of the above nanosilica was also modified using trimethoxy(propyl)silane, by employing an anhydrous surface functionalization route. Under such conditions, silanol production and subsequent reaction are expected to be confined to the vicinity of nanosilica surfaces containing hydroxyl groups/adsorbed water. Detailed information on the surface modification process and the mechanisms involved can be found elsewhere $[43,45]$. This surface modified nanosilica is referred to as C3-treated nanosilica throughout the text.

\subsection{Sample Preparation}

Nanocomposite samples were prepared using a solution blending method, as described in detail elsewhere $[43,45]$. Briefly, the desired amount of nanosilica $(2 \mathrm{wt} \%, 5 \mathrm{wt} \%$ or $10 \mathrm{wt} \%$ ) was sonicated in xylene before the polyethylenes were added. The polyethylene/xylene/silica mixture was then heated to the boiling point of xylene before being poured into cold methanol to induce rapid precipitation. The resultant polyethylene gel containing the entrained nanoparticles was filtered, dried and melt pressed at $150{ }^{\circ} \mathrm{C}$, followed by vacuum drying at $100{ }^{\circ} \mathrm{C}$ for $1 \mathrm{~h}$. For comparison purposes, unfilled polyethylene was processed in the same way as the nanocomposites.

Samples for absorption current and space charge measurements were prepared using a Graseby Specac 25.011 hydraulic press, using a temperature of $150{ }^{\circ} \mathrm{C}$ and a load of 3 ton. The disc so produced was then isothermally crystallized from the melt at $115^{\circ} \mathrm{C}$ for $1 \mathrm{~h}$ in an oil bath (Grant Model W28), followed by quenching into water. All samples were $\sim 200 \mu \mathrm{m}$ in thickness and $30 \mathrm{~mm}$ in diameter. Prior to measurements, all samples were dried in a 
vacuum oven at $60{ }^{\circ} \mathrm{C}$ until no significant weight change could be detected. Samples for absorption current measurements were sputtered coated with gold using an Emitech K550X unit, to ensure good electrical contact between the electrodes and the samples.

\subsection{Electrical Measurements}

Absorption current measurements were performed using a Keithley 6487 picoammeter and a sample holder containing opposing circular electrodes, $20 \mathrm{~mm}$ in diameter. At room temperature, a constant voltage equating to an applied field of either $25 \mathrm{kV}_{(\mathrm{DC})} \mathrm{mm}^{-1}$ or $40 \mathrm{kV}_{(\mathrm{DC})} \mathrm{mm}^{-1}$ was applied and the resulting current was recorded as a function of time, at $5 \mathrm{~s}$ intervals. The pulsed electro-acoustic (PEA) technique [47] was used to determine the space charge behaviour of the material system of interest. A pulse voltage of $600 \mathrm{~V}$ with a pulse width of $5 \mathrm{~ns}$ was employed and silicone oil was used to ensure good acoustic contact between the sample and the electrodes. Calibration was conducted at $10 \mathrm{kV}_{(\mathrm{DC})} \mathrm{mm}^{-1}$ with a short period of voltage application. The evolution of space charge was then determined at room temperature at an applied field of $40 \mathrm{kV}_{(\mathrm{DC})} \mathrm{mm}^{-1}$. The resulting data were processed using the calibration trace and a deconvolution technique, to determine the space charge density as a function of position. LabVIEW software was used for the purpose of data analysis.

\subsection{Results}

\subsection{Dispersion and Morphology}

An in-depth discussion of nanoparticle dispersion and matrix morphology is not the primary emphasis here, since this has been covered elsewhere [43, 45]. Nevertheless, in summary, in all the nanocomposites, a significant mass fraction of the nanofiller appeared in the form of agglomerates, which were not easily broken up and dispersed even after surface modification. This is not uncommon in the context of nanocomposite research [48, 49]. Meanwhile, the morphology of the polymer matrix was perturbed by the inclusion of increasing amount of nanosilica, but such morphological changes have little effect on electrical properties; previous results have clearly shown that variations in space charge behaviour should, primarily, be attributed to the presence of the nanosilica and its surface chemistry, rather than morphological changes in the matrix material [45].

\subsection{Reproducibility of Absorption Current Measurements}


A common issues associated with current measurements through highly insulating materials is a degree of variability in the absolute magnitude of the test data [22]. To address this, Figure 2 shows an example of a plot of repeated absorption current measurements for the case of the unfilled polyethylene; three different pristine samples were used, to eliminate any possible effects associated with measurement history. From these data, it is evident that the absolute magnitude of the absorption current differs from sample to sample and, therefore, this variability provides an estimate of experimental uncertainty in the measured absolute current values. Equivalent repeated experimental runs on all the other material formulations revealed comparable effects and, as such, we conclude that variations less than a factor of 3 in absolute current values are of no statistical significance. However, repeated experimental runs show that the general time dependence of the current is reproducible, as can be seen in Figure 2; similar sample to sample variability was also seen in the nanocomposite systems but these data are not shown here on the grounds of brevity.

\subsection{Absorption Current Measurements at $40 \mathrm{kV}_{(\mathrm{DC})} \mathrm{mm}^{-1}$}

Figure 3 shows plots of the time dependence of absorption current for all the different material formulations considered in this study, all of which were acquired at a constant applied field of $40 \mathrm{kV}_{(\mathrm{DC})} \mathrm{mm}^{-1}$ over $3 \mathrm{~h}$ at room temperature. In the case of the unfilled system, the current falls monotonically over the complete duration of the experiment and, although the rate of decrease of the absorption current within ranges of $10^{0}-10^{3} \mathrm{~s}$ and $10^{3}-10^{4}$ $s$ are not identical, the sample to sample deviations from strict linearity that are evident in Figure 2 indicate that that there is no a priory reason to infer that such fluctuations are significant. However, it is clear from Figure 3 that the nanocomposites exhibit a current-time characteristic that is very different from that of the unfilled polyethylene and that the general form of this is repeatable from sample to sample. That is, the inclusion of the nanofiller has a marked effect on charge transport [50]. In discussing this, we will sub-divide the data into three regimes: Phase I, which corresponds to the initial decrease in current; Phase II, corresponding to the region immediately following the reduction in slope; Phase III, where the current rises. The division of current versus time plots into three distinct regions is somewhat arbitrary, since the transition from one phase to the next is not abrupt. As such, the vertical dashed boundaries indicated in Figure 4 are only intended to be indicative.

Figure 4 presents time dependent absorption current data for the range from 5-200 s. This corresponds to 40 data points and encompasses Phase I. From this, it is qualitatively evident that the rate of decrease of current for all the nanocomposites is significantly greater 
than for the unfilled polyethylene. Although the absolute magnitude of the absorption current varies from sample to sample, the experimental uncertainties in this aspect of behaviour mean that we will not consider this further. In contrast, the rate of decrease of current with time is comparable in all the nanocomposites and repeated experimental runs showed that this is reproducible, as previously highlighted. To evaluate this, each data set was independently fitted to the Curie-von Schweidler Law, using the following procedure. For Phase I, the time dependence of the current was fitted to Equation 1 from an initial time $t_{I i}=5 \mathrm{~s}$ to a final time, $t_{I f}$. By varying $t_{I f}$ and considering the goodness of the fit in terms of both the correlation coefficient and the residuals, $t_{\text {If }}$ could be estimated and, hence, the exponent defining the decay of current in Phase I could be evaluated. While this should produce a reasonable estimate for $t_{I f}$, the quoted uncertainties were derived purely from the statistical analysis of the data points within the temporal region defined as above. As such, they cannot take into account uncertainties in defining transitions from one Phase to the next and, therefore, are likely to represent lower bound values. The lines present in Figure 4 correspond to the resulting fits and the associated Phase I exponent values, $b_{l}$, are listed in Table 1. From this, it is clear that all the nanocomposites exhibit a higher value of the exponent $b_{1}$ that characterizes Phase I, compared with the unfilled polyethylene. Also, this parameter appears to increase somewhat with increasing amount of nanosilica present in the system although, overall, any influence of nanosilica surface modification appears to be less than the experimental uncertainties.

In Phase II, the precise determination of the associated exponent, $b_{2}$, was complicated further by the need to identify both the initial time $t_{I I i}$ and the end point $t_{I I f}$. To address this, the following approach was adopted. First, the Phase I best fit lines were extrapolated into Phase II (shown towards the left of Figure 5) and the point at which this fell significantly below the measured current data was determined for each specimen, to provide an estimate of the onset of Phase II. Since current measurements were made every $5 \mathrm{~s}$ and Phase II typically lasted some $500 \mathrm{~s}$, it was not essential precisely to determine the onset of Phase III in order to generate a reasonable estimate for the exponent $b_{2}$ in Phase II (see Table 2). Nevertheless, as in the case of Phase I, the quoted uncertainties do not take into account the uncertainties inherent in defining both the start and end points of Phase II and, therefore, as above, the values shown represent lower bounds.

Comparing the data presented in Table 1 and Table 2, it is evident that the rate at which the current decreases for all the nanocomposites in Phase II is much lower than in Phase I. This does not apply to the unfilled polyethylene, where the behaviour is best 
described by a single line. While the quantitative value of the exponent $b_{2}$ is not crucial for the argument here, it is worthy of note that, for most nanocomposites, its value is comparable to that which characterizes the unfilled polyethylene.

Using the slope change data in Figure 5, the charge carrier mobility $\mu$ was estimated in each system using Equation 2 and the resulting values are shown in Figure 6. For all the nanocomposites, the derived mobility values fall around $10^{-14} \mathrm{~m}^{2} \mathrm{~V}^{-1} \mathrm{~s}^{-1}$. In the case of the unfilled polymer, no characteristic reduction in slope is evident, indicating that any such transition must occur beyond $10^{4} \mathrm{~s}$. Using a value of $10^{4} \mathrm{~s}$ for the unfilled polymer, $\mu$ is estimated to be $\sim 3 \times 10^{-16} \mathrm{~m}^{2} \mathrm{~V}^{-1} \mathrm{~s}^{-1}$, which represents the upper bound for this parameter. Comparison of this with published data demonstrates that it falls within the measured range for a series of polyethylenes [51], suggesting that the analysis is not unreasonable. Evidently, all the nanocomposites are characterized by a higher charge carrier mobility than the unfilled polyethylene.

In Phase III, the current flowing through the nanocomposites begins to rise and, due to the restrictions inherent in Equation 1, no data analysis comparable to that described above was attempted.

\subsection{Absorption Current Measurements at $25 \mathrm{kV}_{(\mathrm{DC})} \mathrm{mm}^{-1}$}

To confirm the general features described above, the procedure was repeated but using a reduced DC field of $25 \mathrm{kV}_{(\mathrm{DC})} \mathrm{mm}^{-1}$. The resulting data are shown in Figure 7 and, since these are broadly qualitatively consistent with the results shown in Figure 3, they were subjected to the same analysis based upon three regimes. However, at this applied field, the currents that flow are reduced, which makes the measurements more uncertain, and observed variations in behaviour occur over longer time scales. The indicatives dashed lines included in Figure 7 at $300 \mathrm{~s}$, and $3600 \mathrm{~s}$ are intended to indicate the approximate boundaries of Phase I and Phase II respectively, albeit that the relevant values for each data set were established as described above. As a result of the similarities between results obtained at $40 \mathrm{kV}_{(\mathrm{DC})} \mathrm{mm}^{-1}$ and $25 \mathrm{kV}_{(\mathrm{DC})} \mathrm{mm}^{-1}$, only an outline description of the latter data set and the key differences are provided here, for the sake of brevity.

Table 3 summarizes the results obtained at $25 \mathrm{kV}_{(\mathrm{DC})} \mathrm{mm}^{-1}$. In Phase I, the nanocomposites exhibit exponent $b_{1}$ values that are higher than the unfilled polyethylene and, as at $40 \mathrm{kV}_{(\mathrm{DC})} \mathrm{mm}^{-1} ; b_{1}$ again seems to increase with increased nanosilica loading level and, again, the effect on this parameter of nanosilica surface modification falls within the 
experimental uncertainties, which are greater here than at $40 \mathrm{kV}_{(\mathrm{DC})} \mathrm{mm}^{-1}$ due to the reduced currents that flow at the lower applied field. In Phase II, the estimated values of $b_{2}$ for the nanocomposites are generally lower than the invariant exponent deduced for the unfilled polyethylene, suggesting that the similarity in the $b_{2}$ values obtained from the unfilled polymer and the nanocomposites at $40 \mathrm{kV}_{(\mathrm{DC})} \mathrm{mm}^{-1}$ was coincidental. Mobility values deduced from all the materials are comparable with those shown in Figure 6; in the nanocomposites, the mobility falls around $\sim 3 \times 10^{-14} \mathrm{~m}^{2} \mathrm{~V}^{-1} \mathrm{~s}^{-1}$ while, for the unfilled polymer, the value appears about $1 \frac{1}{2}$ orders of magnitude lower.

\subsection{Space Charge Dynamics}

In previous work, the space charge behaviour of the same materials as considered here has been reported over a period of $1 \mathrm{~h}$ [45]. Briefly, in the case of the unfilled polymer, the extent of space charge accumulation was considered insignificant. In contrast, in the nanocomposites, homocharge was found to develop near both the anode and cathode and then move into the sample bulk with increasing time, in line with the data shown in Figure 8a. It is noteworthy that increasing the nanofiller loading level resulted in the accumulation of increased quantities of homocharge near both electrodes, for both the nanocomposites containing the untreated and C3-treated nanosilica. Meanwhile, the accumulation of space charge in the nanocomposites containing the $\mathrm{C} 3$-treated nanosilica was less than that in the nanocomposites containing an equivalent amount of the untreated nanofiller. Nevertheless, the space charge dynamics in all the nanocomposites revealed a similar pattern as a function of time.

Considering the similarities in the space charge trend for all the nanocomposites within $1 \mathrm{~h}$, the effect of space charge in a representative set of the nanocomposites, i.e., the nanocomposites containing the untreated nanosilica, was studied for a much longer duration, i.e., up to $8 \mathrm{~h}$, at an applied field of $40 \mathrm{kV}_{(\mathrm{DC})} \mathrm{mm}^{-1}$. Over this extended period, the magnitude of the homocharge initially increased adjacent to the electrodes (see Figure 8a) and, then, as the charge moved into the sample bulk, progressively decreased (see Figure 8b). This was followed by the development of heterocharge near the cathode, while the magnitude of homocharge near the anode remained constant (see Figure 8c). The change from homocharge to heterocharge near the cathode is likely to have a marked effect on charge injection processes and, consequently, should affect many different electrical factors [52, 53]. Indeed, charge packets are evident in Figure 8, despite the relatively low field. The profile of space charge in the nanocomposites containing the $\mathrm{C} 3$-treated nanosilica is expected to mirror 
that in the nanocomposites containing the untreated nanosilica, but with less apparent charge development effects. Since the development of space charge in the unfilled polyethylene was considered insignificant in comparison with the nanocomposites [45], no similar long-term measurements were attempted.

\subsection{Discussion}

From the data presented above, it is evident that all the nanocomposites, irrespective of nanofiller loading level or surface chemistry, are characterized by a very different currenttime behaviour compared with the unfilled polyethylene. In Phase I, the time dependence of the absorption current in the nanocomposites is characterized by an exponent $b_{1}$, which is of the order of 0.5 . Wintle [54] has indicated that $0 \leq b_{n} \leq 2$ is consistent with dipole orientation, carrier tunnelling and carrier hopping, while $0 \leq b_{n} \leq 1$ is consistent with charge injection forming trapped space charge. Roy [28] reported that the absorption current behaviour was independent of electrode materials and sample thickness and suggested that electrode polarization and dipole orientation were unlikely to be the reason for the absorption current, which is in line with the work of Das-Gupta and Brockley [50]. As such, we propose that the $b_{n}$ values observed in our case are largely determined by charge trapping processes.

From above, the $b_{1}$ values obtained from the nanocomposites are significantly higher than for the unfilled polyethylene, which implies that additional charge trapping processes occur within the nanocomposites, presumably due to interfacial trapping sites. Such interfacial trapping sites may serve either to inhibit charge transport through the system (i.e. if the associated trap states lie at a low energy - deep traps) or enhance it (shallow traps, particularly if closely spaced) [55]. The change in the $b_{n}$ exponent seen in all the nanocomposites on entering Phase II can be interpreted in a number of ways. First, it could indicate a change in the dominant absorption current mechanism, which may be related to interfacial polarization effects, as proposed elsewhere [28, 56]. Second, Smith [24] suggested that the mobility of charge carriers could be highly dependent upon the trapping and release of charges from deep traps, in addition to the underlying current associated with the large number of shallower traps present within the system. That is, as the experiment progresses, the distribution of vacant deep trapping sites varies. Although the increasing absorption current with poling time seen in Phase III is highly unusual, such observations are not without precedent. A comparable increase of current was previously reported by Smith [24] for microcomposites and, although it was suggested that this may in some way be related to polarization effects at the filler / matrix interface, no convincing explanation was proposed. 
The above discussion has focussed exclusively on processes that occur throughout the bulk. However, space charge data obtained over a long duration indicate that variations in the space charge distributions adjacent to the electrodes may also play an important part in explaining the behaviour of the nanodielectrics. From data such as those presented in Figure 8 , it is evident that homocharge initially develops near both the anode and the cathode, which will serve to reduce the electric field at the electrodes and thereby progressively reduce charge injection into the material. This may well explain the higher $b_{1}$ values seen in the nanocomposites than in the unfilled polyethylene, where no appreciable space charge developed over comparable timescales [45]. With increasing time, the quantity of homocharge from both electrodes increases and the charge moves progressively towards the sample bulk. However, as discussed above for Phase II, charge carriers may move rapidly through the system for a short time, but the effective mobility may be greatly reduced as a consequence of being immobilized for a much longer period in localized, low lying states. After $3 \mathrm{~h}$ of voltage application, homocharge from both electrodes starts to overlap (see Figure $8 b$ ) and, moreover, heterocharge begins to develop near the cathode, while the homocharge near the anode remains constant. This cathode heterocharge will result in a marked increase in the local field intensity and increased charge injection; the reversal of the dominant local space charge from homocharge to heterocharge near the cathode may then contribute to the increase in current seen in Phase III.

Although the above discussion of experimental data is entirely qualitative, it is complemented by the quantitative modelling work of Anta et al. [57], who considered the effect of trap depth on charge transport through polyethylene; shallow traps were considered to arise from physical or conformational defects while, in their unfilled system, deep traps were associated with chemical defects or molecular impurities. This work set out to evaluate the current-voltage characteristics of polyethylene from the density of localized states, where charge transport was modelled as a thermally activated multiple trapping/detrapping process. The work showed that electron mobility is a strong function of the electron density and since the residence time of electrons in deep traps is much longer than those in shallow traps, the net mobility of the electrons increases as the electron density in the system increases, in line with the well-known trap filling effect. Although the above analysis was considered in terms of steady state conduction at different fields, it would appear to have parallels with the dynamic case described above. Indeed, Anta et al. [57] relate their findings to complementary experimental work [58] describing the onset of charge packet formation at 
very high fields $\left(>60 \mathrm{kV}_{(\mathrm{DC})} \mathrm{mm}^{-1}\right)$. From Figure 8, it would appear that charge packets are able to develop at this much lower field, albeit over long timescales.

We therefore propose that charge transport behaviour shown in Figure 8 for the nanocomposites is initially characterized by trap-limited conduction and may be dominated by trap-limited ohmic conduction in addition to trap-limited SCLC (see Equation 3); this characterizes Phase I. As the voltage continues to be applied (but not increased), deep traps are progressively filled; Takada et al. [59] have proposed that the trapping sites associated with nanoparticles may be very deep $(1.5-5 \mathrm{eV})$ and residence times in deep traps have been proposed by Munier et al. [41] that are comparable to our total experimental duration. In Phase II, the influence of the filled traps becomes significant, such that the absorption current behaviour in Phase II is different from that in Phase I. Nevertheless, we suggest that Phase II is still characterized by trap-limited conduction, but is now dominated by trap-limited SCLC. When all the deep traps are filled and the trap-filled limit is reached, an increase in current results and this combined with increased charge injection from the cathode as a result of the increasing cathode heterocharge underlies the behaviour seen in Phase III. This qualitative analysis has much in common with the numerical simulation of space charge accumulation and conduction in nanocomposites described by Min et al. [44], who highlight the importance of charge trapping in affecting both charge injection into nanodielectrics and subsequent charge transport dynamics in the bulk. Indeed, Figure 3 in [44], which presents the time dependent external current densities calculated for nanodielectrics containing different trap densities, shows, for some systems, a relatively rapid initial decrease in current density (up to about $100 \mathrm{~s}$ ), followed by a regime in which the rate of decrease of current density is reduced $\left(10^{2}-10^{4} \mathrm{~s}\right)$, after which, the current density increases.

\subsection{Conclusions}

The emergence of nanodielectrics has seen many unique electrical properties reported, mainly ascribed to the presence of the nanofiller/polymer interfaces. In our case, while the current flowing through the unfilled polyethylene decreased with time in a conventional manner, all the investigated nanocomposites exhibited a current-time characteristic that can be considered in terms of three phases: the region corresponding to the initial decrease of current (Phase I), in which the rate of decrease of current for all the nanocomposites is significantly greater than for the unfilled polyethylene; the region immediately following the initial reduction in slope (Phase II), which was not evident for the unfilled polyethylene; and the region where the current rises (Phase III), not applicable for the unfilled polyethylene. In 
this, we suggest the possibility of increased charge trapping mechanisms in the nanocomposites due to the presence of the nanofiller/polymer interface. Meanwhile, our results from space charge measurements showed that homocharge started to develop near both the anode and the cathode of the nanocomposites at the beginning of the test (no appreciable space charge development was found in the unfilled polyethylene over comparable timescales), and this may explain the rapid decrease of current in the nanocomposites in Phase I, as compared with the unfilled polyethylene. Continuous homocharge accumulation leads to the interpretation that, in Phase II, the effective mobility may be greatly reduced as a consequence of charge carriers being immobilized for a much longer period in localized states. Thereafter, the development of heterocharge near the cathode (a reversal from the initial homocharge development) possibly leads to the increase in current in Phase III. We subsequently suggest the presence of SCLC and its trap-filled limit in the nanocomposites.

\section{Acknowledgments}

The authors acknowledge the Ministry of Higher Education, Malaysia and Universiti Teknologi Malaysia for financial sponsorship and the research votes 4F707 and 11J41. All data supporting this study are openly available from the University of Southampton repository at http://dx.doi.org/10.5258/SOTON/386426.

\section{References}

[1] Fan B, and Bai J 2015 J. Phys. D: Appl. Phys. 48455303

[2] Zhu J, Li W, Huo X, Li L, Li Y, Luo L and Zhu Y 2015 J. Phys. D: Appl. Phys. 48355301

[3] Diaham S, Saysouk F, Locatelli M-L and Lebey T 2015 J. Phys. D: Appl. Phys. 48385301

[4] Virtanen S, Ranta H, Ahonen S, Karttunen M, Pelto J, Kannus K and Pettersson M 2014 J. Appl. Polym. Sci. 13139504

[5] Lewis T J 2004 IEEE Trans. Dielectr. Electr. Insul. 11739

[6] Tanaka T, Kozako M, Fuse N and Ohki Y 2005 IEEE Trans. Dielectr. Electr. Insul. 12669

[7] Raetzke S and Kindersberger J 2010 IEEE Trans. Dielectr. Electr. Insul. 17607

[8] Lau K Y, Vaughan A S and Chen G 2015 IEEE Electr. Insul. Mag. 3145 
[9] Lewis T J 2005 J. Phys. D: Appl. Phys. 38202

[10] Kozako M, Fuse N, Ohki Y, Okamoto T and Tanaka T 2004 IEEE Trans. Dielectr. Electr. Insul. 11833

[11] Nelson J K and Fothergill J C 2004 Nanotechnology 15586

[12] Tanaka T, Montanari G C and Mülhaupt R 2004 IEEE Trans. Dielectr. Electr. Insul. 11763

[13] Roy M, Reed C W, MacCrone R K, Schadler L S, Nelson J K, Keefe R and Zenger W 2005 Proc. IEEE Int. Symp. Electrical Insulating Materials (Kitakyushu, Japan) 223

[14] Roy M, Nelson J K, MacCrone R K, Schadler L S, Reed C W, Keefe R and Zenger W 2005 IEEE Trans. Dielectr. Electr. Insul. 12629

[15] Montanari G C, Palmieri F, Testa L, Motori A, Saccani A and Patuelli F 2006 IEEJ Trans. Fundam. Mater. 1261090

[16] Green C D, Vaughan A S, Mitchell G R and Liu T 2008 IEEE Trans. Dielectr. Electr. Insul. 15134

[17] Lewis T J 1994 IEEE Trans. Dielectr. Electr. Insul. 1812

[18] Cao Y, Irwin P C and Younsi K 2004 IEEE Trans. Dielectr. Electr. Insul. 11797

[19] Chen G, Zhang C and Stevens G 2007 IEEE Annual Report Conference on Electrical Insulation and Dielectric Phenomena (Vancouver, Canada) pp 275

[20] Ieda M 1977 IEEE Trans. Electr. Insul. 22261

[21] Fothergill J C 2010 Electrical Properties Dielectric Polymer Nanocomposites ed J K Nelson (New York: Springer) pp 1-30

[22] Adamec V and Calderwood J H 1978 J. Phys. D: Appl. Phys. 11781

[23] Morin P, Alquie C and Lewiner J 1994 Proc. IEEE Int. Sypm. Electrets (Paris, France) pp 265

[24] Smith R C 2009 Mechanistic Electrical Behaviour of Crosslinked Polyethylene / Silica Nanocomposites Ph.D. Thesis Rensselaer Polytechnic Institute

[25] Das Gupta D K and Joyner K 1976 J. Phys. D: Appl. Phys. 9829

[26] Guo T C and Guo W W 1983 J. Phys. C: Solid St. Phys. 161955

[27] Mazur K 1995 Proc. IEEE Int. Conf. Conduction and Breakdown in Solid Dielectrics (Leicester, UK) 98

[28] Roy M 2005 An Examination of the Potential for Nano-composites in the Formulation of HV Cable Insulation Ph.D. Thesis Rensselaer Polytechnic Institute

[29] Many A and Rakavi G 1962 Physical Review 1261980

[30] Malec D, Truong V H, Essolbi R and Hoang T G 1998 IEEE Trans. Dielectr. Electr. Insul. 5 301

[31] Smith R C, Liang C, Landry M, Nelson J K and Schadler L S 2008 IEEE Trans. Dielectr. Electr. Insul. 15187 
[32] Dissado L A and Fothergill J C 1992 Charge Injection and Transport in Insulating Polymers (Electrical Degradation and Breakdown in Polymers vol 9) ed G C Stevens (London: The Institution of Engineering and Technology) chapter 9207

[33] Montanari G C 2000 IEEE Trans. Dielectr. Electr. Insul. 3309

[34] Zhang P, Li G, Gai L and Lei Q 2006 Proc. IEEE Int. Conf. Properties and Applications of Dielectric Materials (Bali, Indonesia) 755

[35] Castellon J, Nguyen H N, Agnel S, Toureille A, Frechette M, Savoie S, Krivda A and Schmidt L E 2011 IEEE Trans. Dielectr. Electr. Insul. 18651

[36] Tian F, Lei Q, Wang Z and Wang Y 2012 IEEE Trans. Dielectr. Electr. Insul. 19763

[37] Mott N F and Gurney R W 1940 Electronic Processes in Ionic Crystals (London: Oxford University Press)

[38] Mazzanti G and Marzinotto M 2013 Space Charge in HVDC Extruded Insulation: Storage, Effects and Measurement Methods (Extruded Cables for High-Voltage Direct-Current Transmission: Advances in Research and Development) (USA: John Wiley and Sons) chapter 499

[39] Wang W, Min D and Li S 2016 IEEE Trans. Dielectr. Electr. Insul. 23564

[40] Wang Y, MacKernan D, Cubero D, Coker D F and Quirke N 2014 J. Chem. Phys. 140 154902

[41] Meunier M, Quirke N and Aslanides A 2001 J. Chem. Phys. 1152876

[42] Shi N and Ramprasad R 20082011 IEEE Trans. Dielectr. Electr. Insul. 15170

[43] Lau K Y, Vaughan A S, Chen G, Hosier I L and Holt A F 2013 J. Phys. D: Appl. Phys. 46 095303

[44] Min, D; Wang, W; Li, S 2015 IEEE Trans. Dielectr. Electr. Insul. 221483

[45] Lau K Y, Vaughan A S, Chen G, Hosier I L, Holt A F and Ching K Y 2014 IEEE Trans. Dielectr. Electr. Insul. 201421340

[46] Lau K Y, Vaughan A S, Chen G, Hosier I L and Holt A F 2013 J. Phys.: Conf. Series. 472 012003

[47] Takada T and Sakai T 1983 IEEE Trans. Electr. Insul. 18619

[48] Kontou E and Niaounakis M 2006 Polymer 471267

[49] Tanaka T et al. 2011 IEEE Trans. Dielectr. Electr. Insul. 18 1484-517

[50] Das Gupta D K and Brockley R S 1978 J. Phys. D.: Appl. Phys. 11955

[51] Montanari G C, Mazzati G, Palmieri F, Motori A, Perego G and Serra S 2001 J. Phys. D.: Appl. Phys. 34 2902-11

[52] Roy M, Nelson J K, MacCrone R K and Schadler L S 2007 J. Mater. Sci. 423789

[53] Baudoin F, Mills D H, Lewin P L, Le Roy S, Teyssedre G, Laurent C and Clain S $2012 \mathrm{~J}$. Phys. D: Appl. Phys. 45325303 (11pp) 
[54] Wintle H J 1983 Conduction processes in polymers Engineering Dielectrics vol IIA ed R Bartnikas and R M Eichhorn (Philadelphia: ASTM) 239

[55] Fleming R J 2010 The emerging mechanistic picture Dielectric Polymer Nanocomposites ed J K Nelson (New York: Springer) 285

[56] Roy M, Nelson J K, MacCrone R K and Schadler L S 2007 J. Mater. Sci. 423789

[57] Anta J A, Marcelli G, Meunier M and Quirke N 2002 J. Appl. Phys. 921002

[58] Teyssedre G, Laurent C, Montanari G C, Palmieri F, See A, Dissado L A and Fothergill J C 2001 J. Phys. D.: Appl. Phys. 342830

[59] Takada T, Hayase Y, Tanaka Y and Okamoto T 2008 IEEE Trans. Dielectr. Electr. Insul. 15 152 


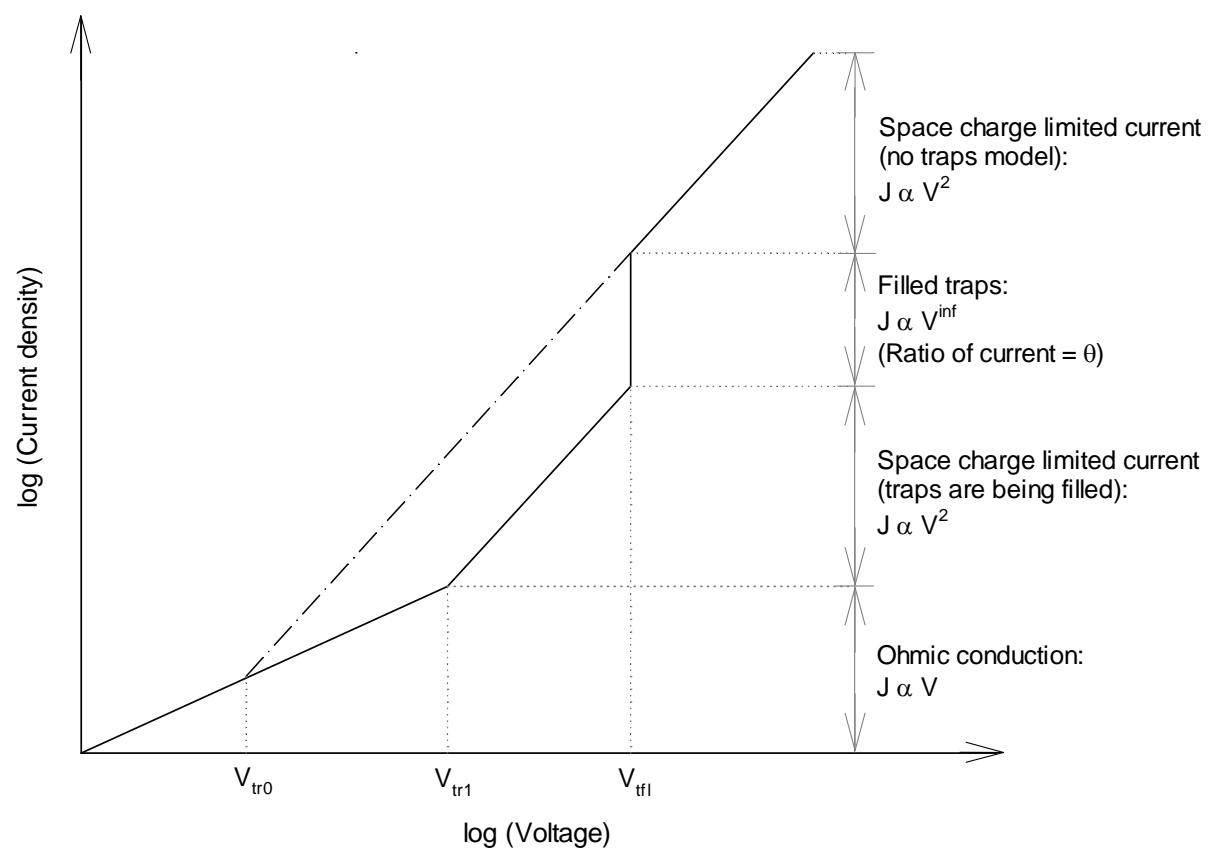

Figure 1. Schematic plot showing current density as a function of applied voltage. 


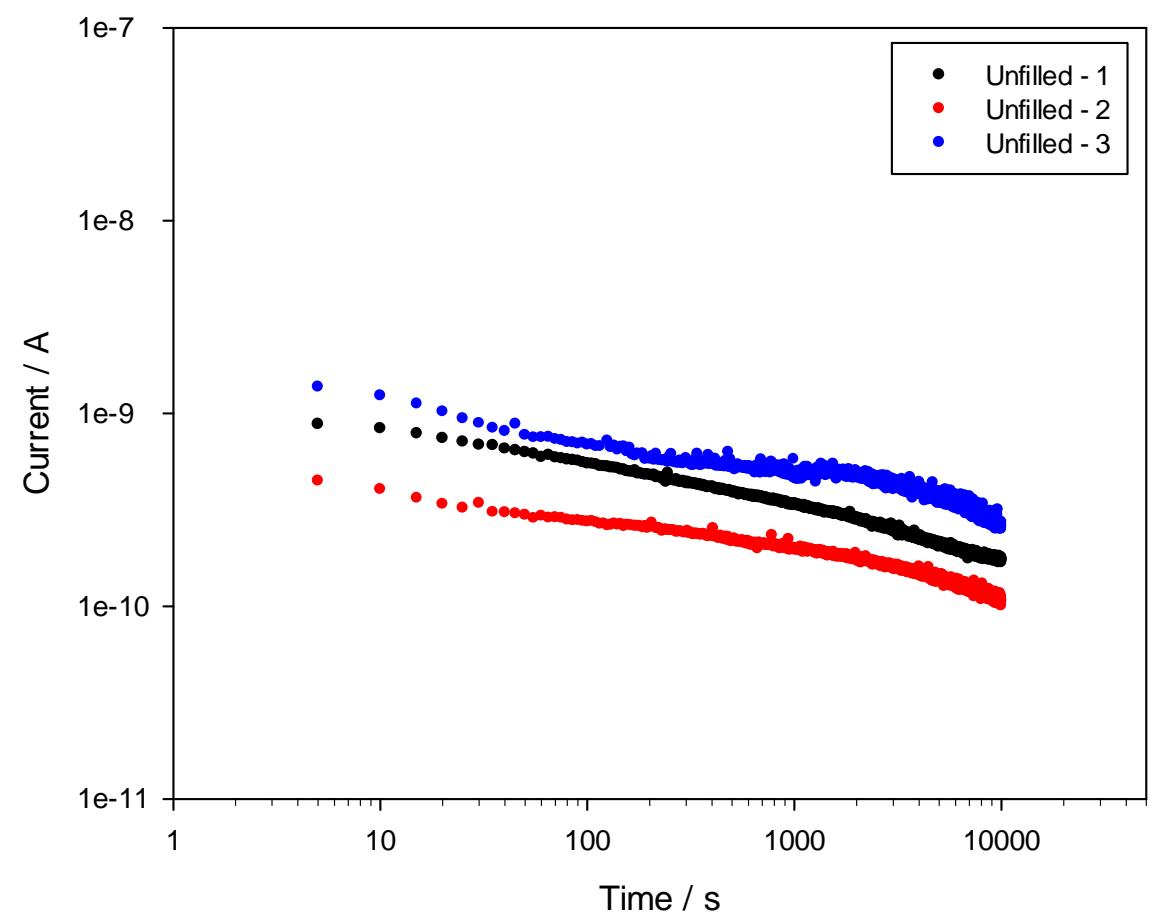

Figure 2. Plots showing repeated measurements of absorption current at an applied field of $40 \mathrm{kV}_{(\mathrm{DC})} \mathrm{mm}^{-1}$ against time up to $10^{4} \mathrm{~s}$ for unfilled polyethylene samples crystallized isothermally at $115^{\circ} \mathrm{C}$. Three different, pristine samples were used to eliminate any possible effects associated with measurement history. 


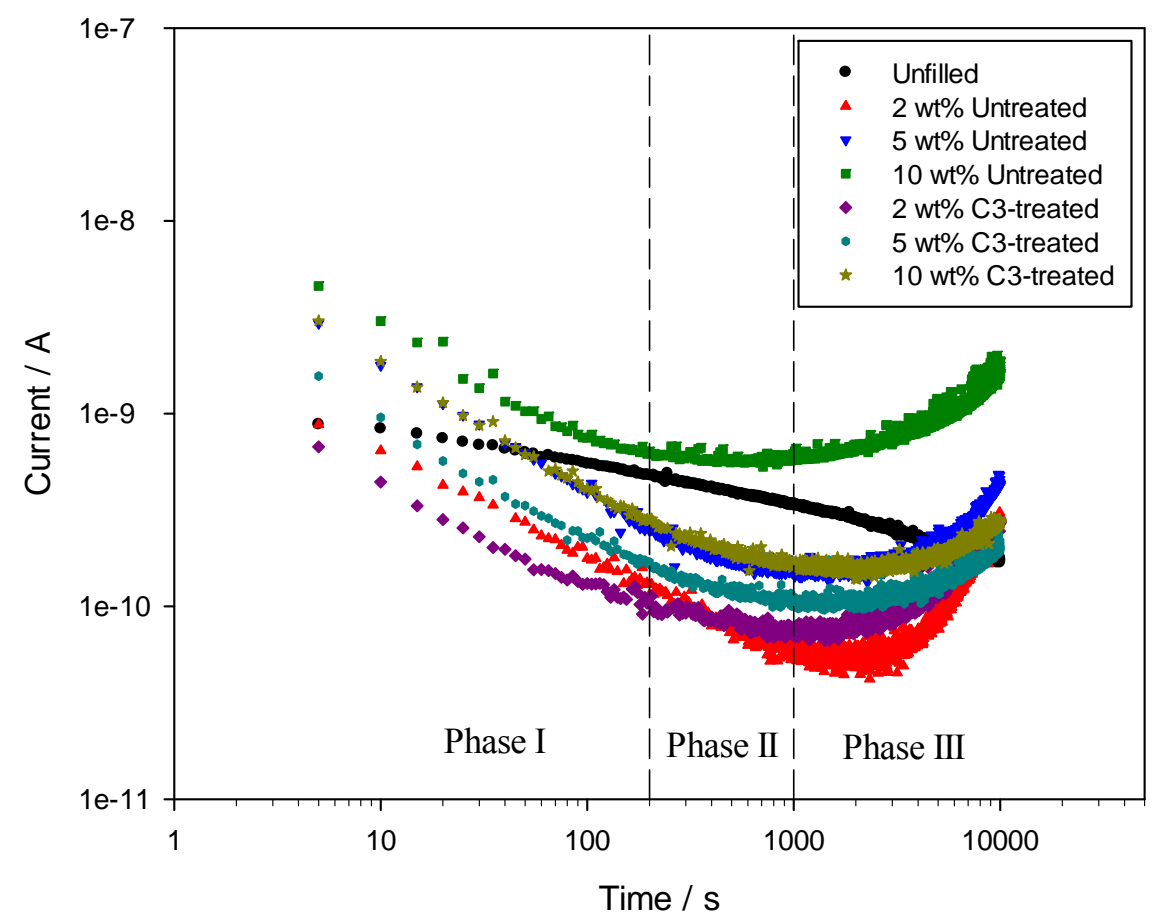

Figure 3. Plots of absorption current at an applied field of $40 \mathrm{kV}_{(\mathrm{DC})} \mathrm{mm}^{-1}$ against time up to $10^{4} \mathrm{~s}$ for all investigated samples crystallized isothermally at $115^{\circ} \mathrm{C}$. The data were divided into three phases for ease of interpretation. 


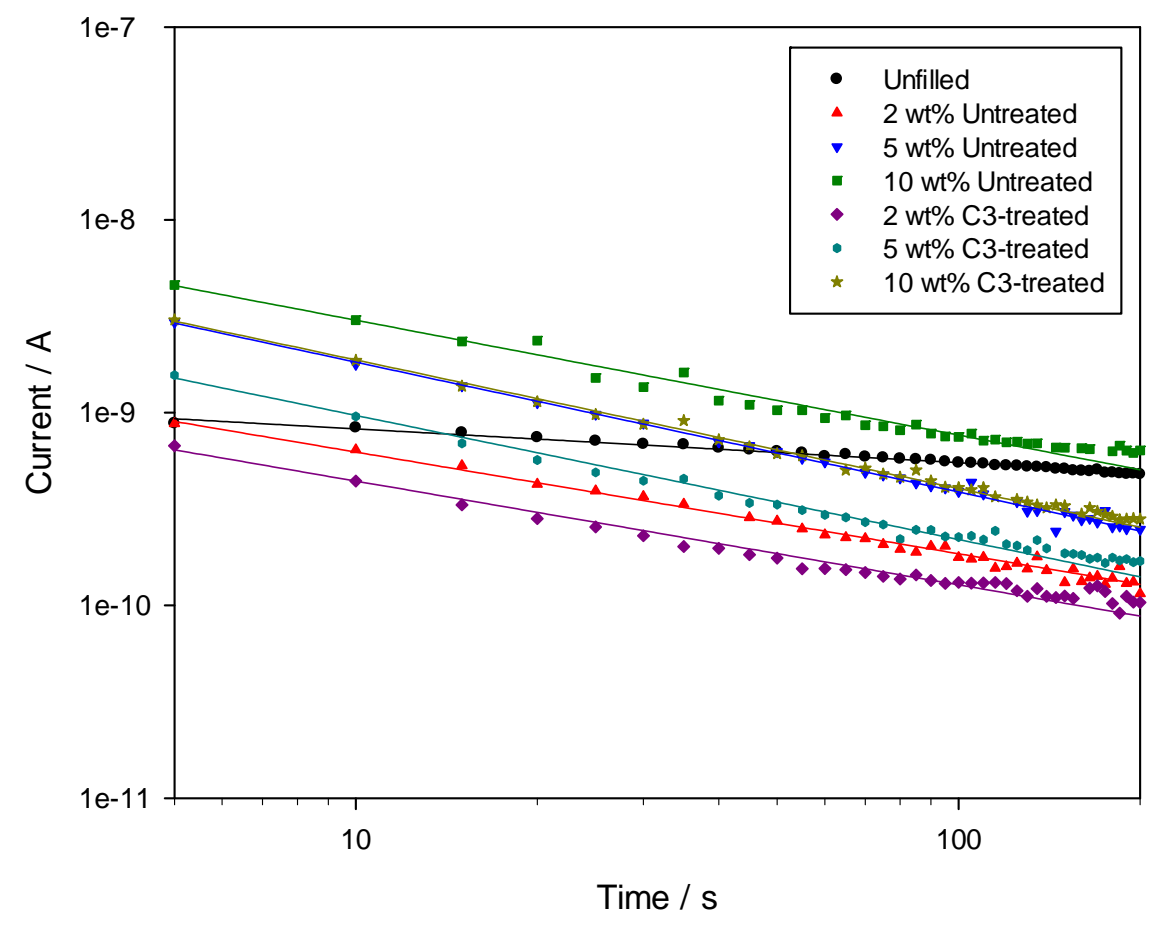

Figure 4. Comparison of experimental data and a power law line fitting for absorption current data up to $200 \mathrm{~s}$. In all cases, the rate of decrease of current with time is greater in the nanocomposites than in the unfilled polyethylene. All data were acquired at an applied field of $40 \mathrm{kV}_{(\mathrm{DC})} \mathrm{mm}^{-1}$. 


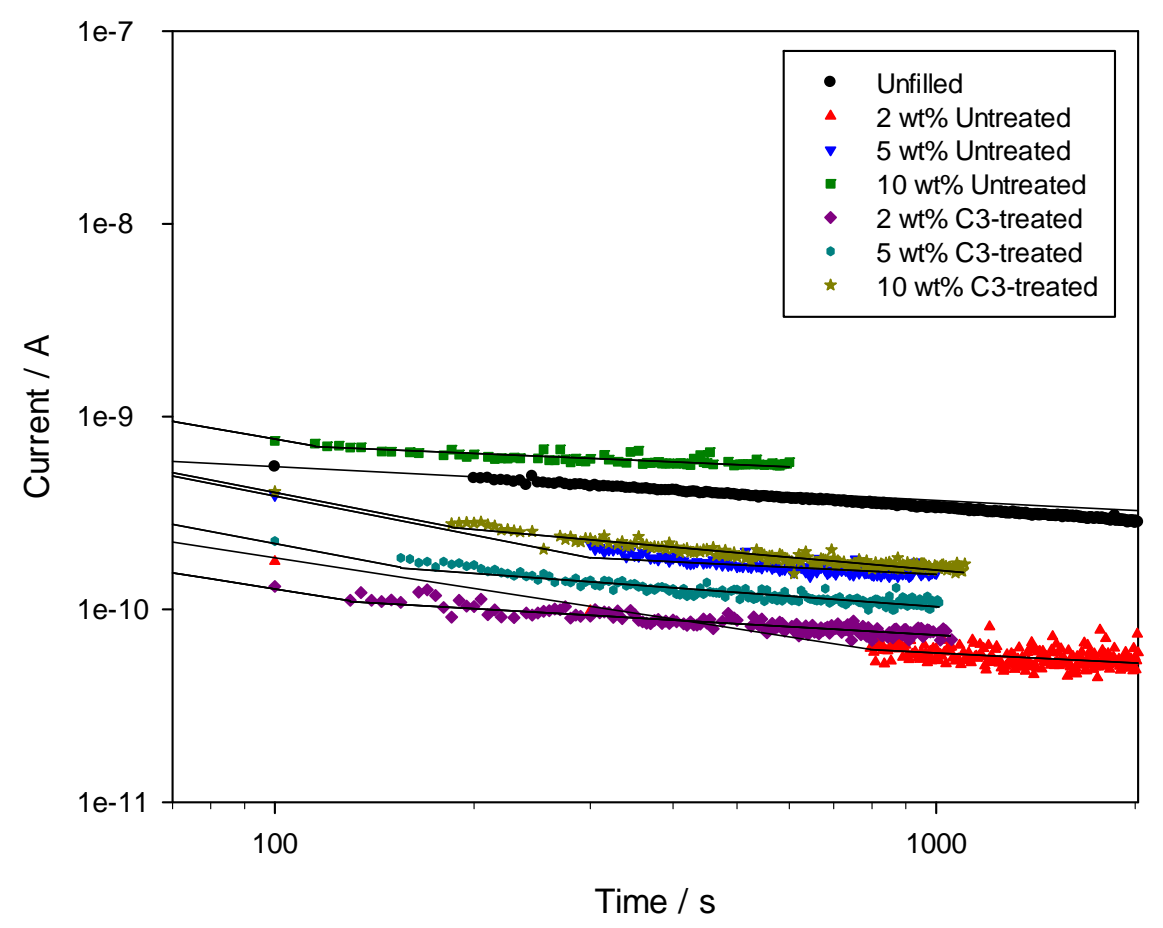

Figure 5. Comparison of experimental data and power law line fitting for absorption current data for Phase II at an applied field of $40 \mathrm{kV}_{(\mathrm{DC})} \mathrm{mm}^{-1}$. Fitted lines from Phase I are also shown, to indicate the point at which a change of slope occurs in nanocomposites. All data were acquired at an applied field of $40 \mathrm{kV}_{(\mathrm{DC})} \mathrm{mm}^{-1}$. 


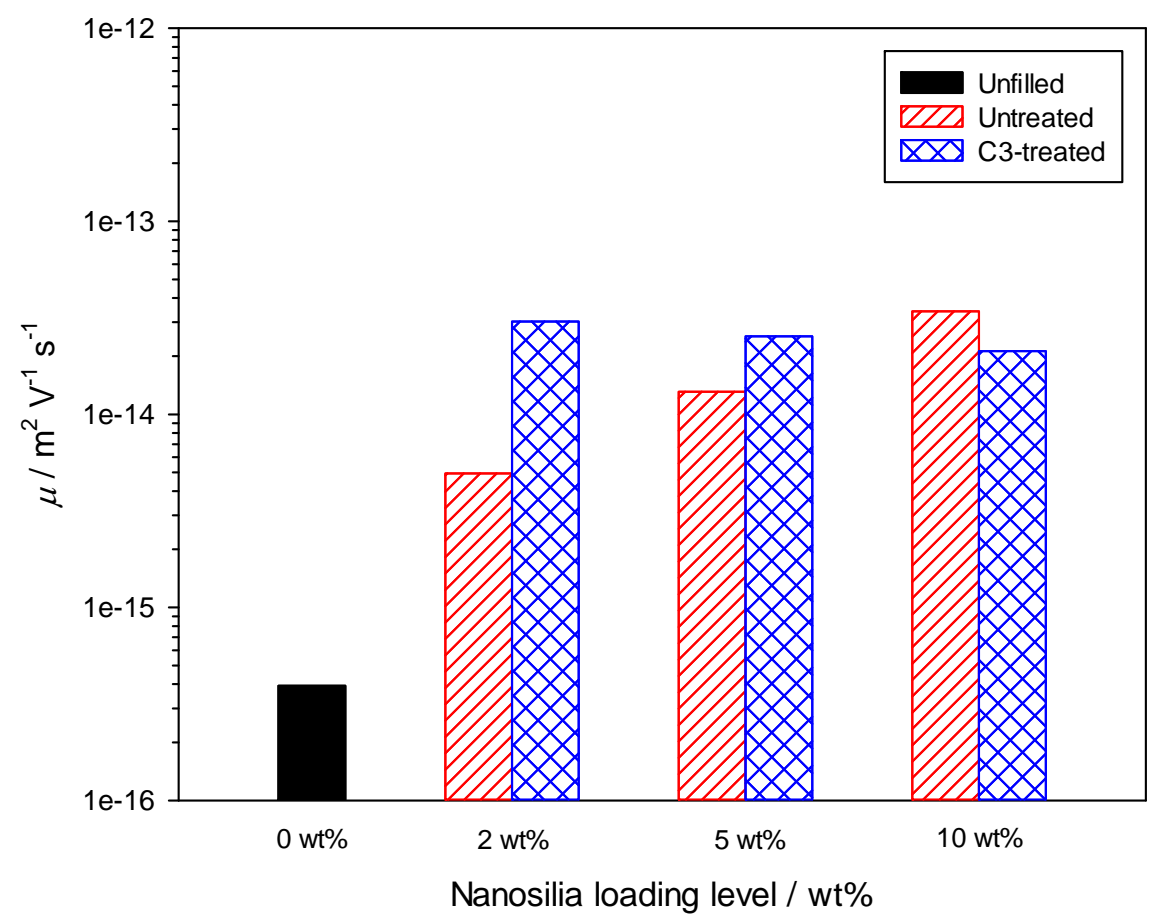

Figure 6. Charge carrier mobility of unfilled polyethylene and nanocomposites containing different types and amounts of nanosilica, obtained from an applied field of $40 \mathrm{kV}_{(\mathrm{DC})} \mathrm{mm}^{-1}$. 


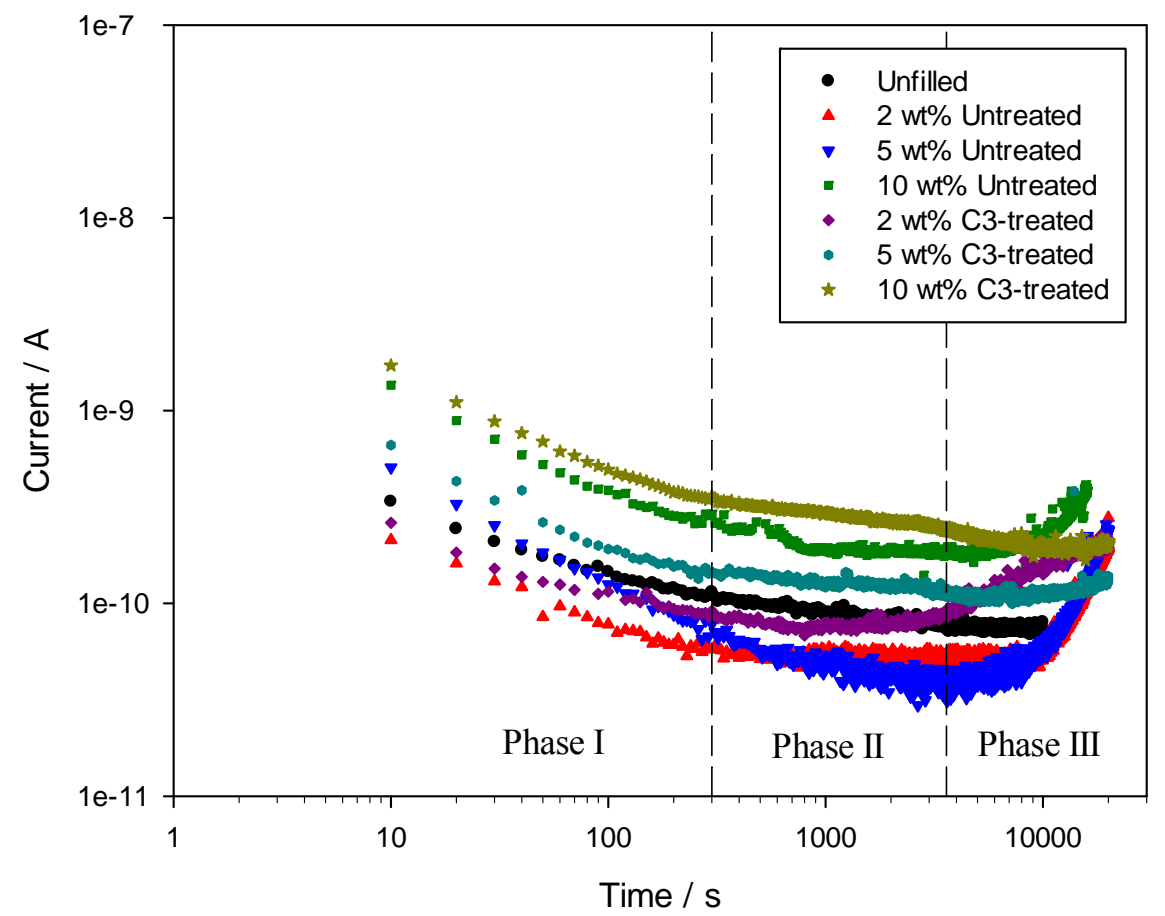

Figure 7. Plots of absorption current against time for all samples crystallized isothermally at $115^{\circ} \mathrm{C}$ at an applied field of $25 \mathrm{kV}_{(\mathrm{DC})} \mathrm{mm}^{-1}$ up to $\left(2 \times 10^{4}\right) \mathrm{s}$. 
(a)

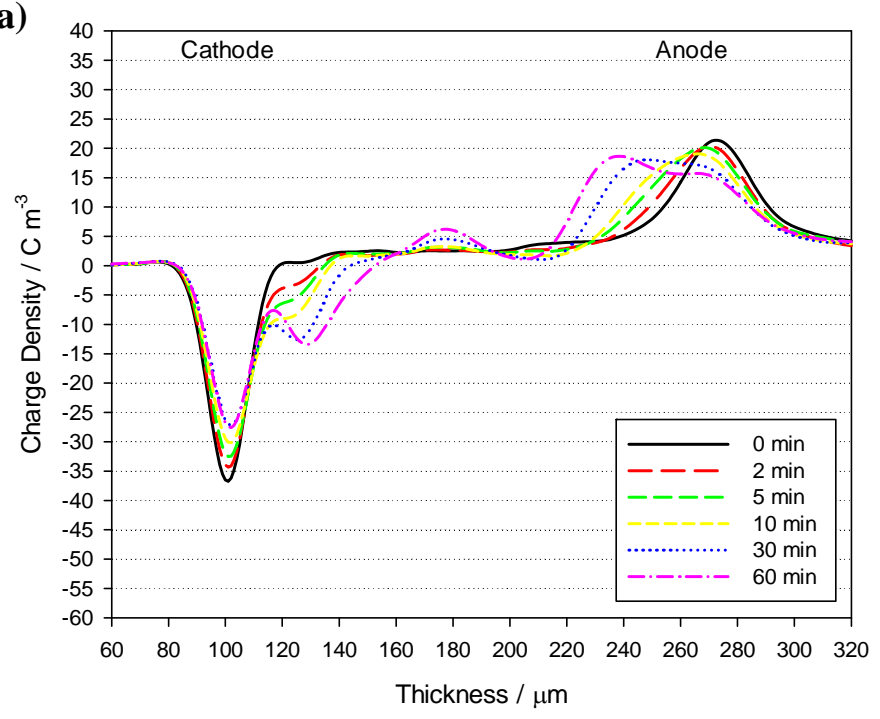

(b)

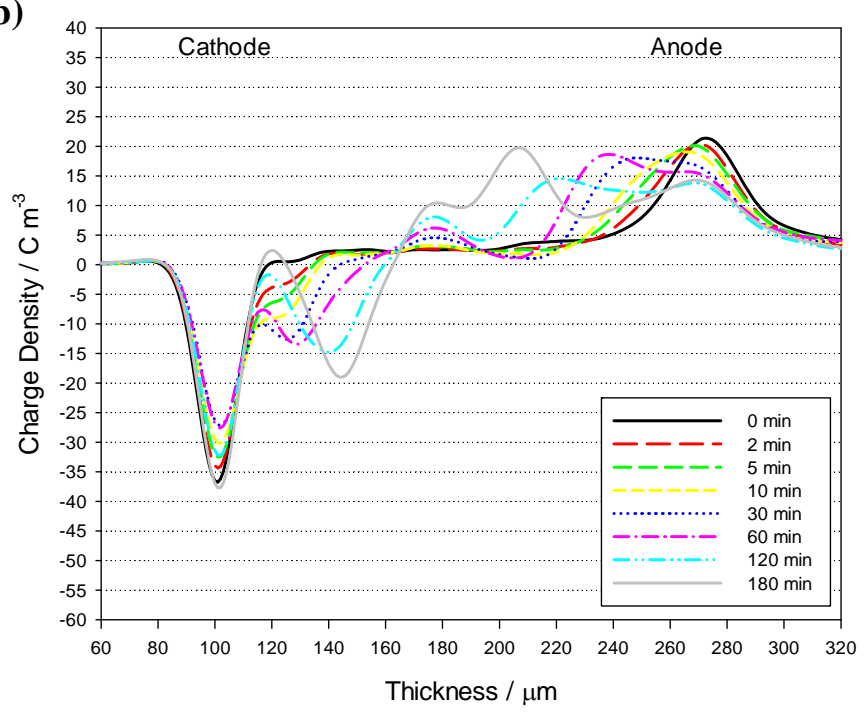

(c)

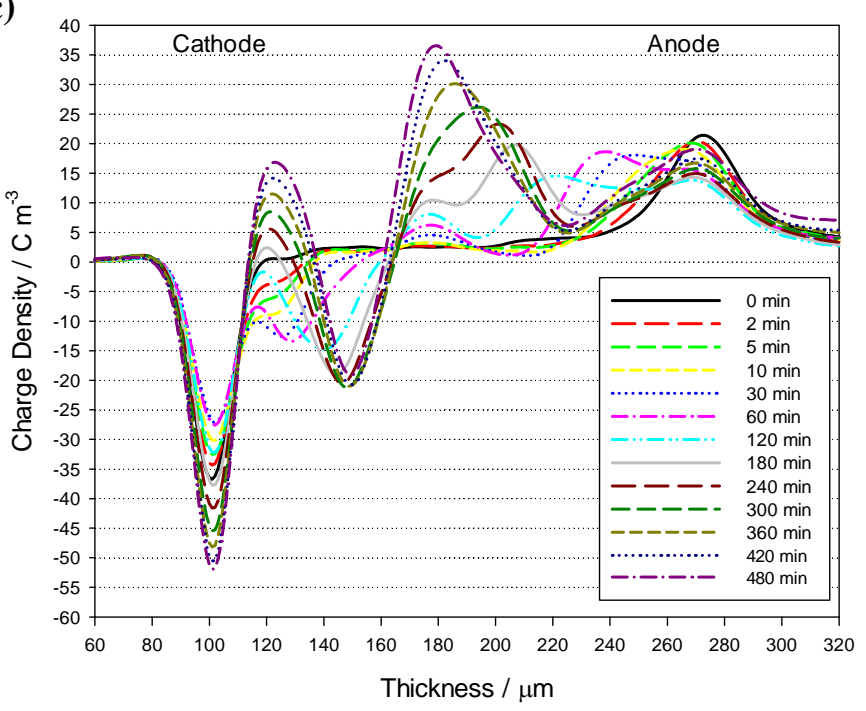

Figure 8. Space charge behaviour of nanocomposites containing $5 \mathrm{wt} \%$ of untreated nanosilica stressed at $40 \mathrm{kV}_{(\mathrm{DC})} \mathrm{mm}^{-1}$ for a typical period of (a) $1 \mathrm{~h}$ and an extended period of (b) $3 \mathrm{~h}$, (c) $8 \mathrm{~h}$. 
Table 1. Exponent values obtained by fitting time dependent absorption current data to the Curie-von Schweidler Law over the time period 5-200 s at an applied field of $40 \mathrm{kV}_{(\mathrm{DC})} \mathrm{mm}^{-1}$.

\begin{tabular}{ll}
\hline Sample & $\boldsymbol{b}_{\mathbf{1}} \times \mathbf{1 0}^{-\mathbf{2}}$ \\
\hline Unfilled & $17.5 \pm 0.3$ \\
$2 \mathrm{wt} \%$ Untreated & $52.7 \pm 0.6$ \\
$5 \mathrm{wt} \%$ Untreated & $67.3 \pm 0.4$ \\
$10 \mathrm{wt} \%$ Untreated & $59.6 \pm 1.3$ \\
$2 \mathrm{wt} \% \mathrm{C} 3$-treated & $53.9 \pm 1.0$ \\
$5 \mathrm{wt} \% \mathrm{C} 3$-treated & $64.4 \pm 0.9$ \\
$10 \mathrm{wt} \% \mathrm{C} 3$-treated & $66.7 \pm 0.6$ \\
\hline
\end{tabular}


Table 2. Exponent values calculated from the absorption current data in Phase II at an applied field of $40 \mathrm{kV} \mathrm{mm}^{-1}$.

\begin{tabular}{ll}
\hline Sample & $\boldsymbol{b}_{\mathbf{2}} \times \mathbf{1 0}^{-\mathbf{2}}$ \\
\hline Unfilled & $17.5 \pm 0.3$ \\
$2 \mathrm{wt} \%$ Untreated & $17.3 \pm 2.0$ \\
$5 \mathrm{wt} \%$ Untreated & $16.5 \pm 2.4$ \\
$10 \mathrm{wt} \%$ Untreated & $14.6 \pm 1.3$ \\
$2 \mathrm{wt} \% \mathrm{C} 3$-treated & $19.6 \pm 0.9$ \\
$5 \mathrm{wt} \% \mathrm{C} 3$-treated & $24.7 \pm 0.8$ \\
$10 \mathrm{wt} \% \mathrm{C} 3$-treated & $30.2 \pm 0.8$ \\
\hline
\end{tabular}


Table 3. Calculated exponent values for Phases I and II at an applied field of $25 \mathrm{kV} \mathrm{mm}^{-1}$, plus associated estimates of charge carrier mobility.

\begin{tabular}{lccc}
\hline Sample & $\boldsymbol{b}_{\mathbf{1}} \times \mathbf{1 0}^{-2}$ & $\boldsymbol{b}_{2} \mathbf{\times} \mathbf{1 0}^{\mathbf{2}}$ & $\boldsymbol{\mu} / \boldsymbol{m}^{2} \boldsymbol{V}^{\mathbf{1}} \mathbf{s}^{-\mathbf{1}}$ \\
\hline Unfilled & $21.0 \pm 0.4$ & $21.0 \pm 0.4$ & $6.3 \times 10^{-16}$ \\
$2 \mathrm{wt} \%$ Untreated & $41.3 \pm 1.1$ & $2.8 \pm 0.4$ & $2.7 \times 10^{-14}$ \\
$5 \mathrm{wt} \%$ Untreated & $59.0 \pm 0.6$ & $22.2 \pm 0.9$ & $2.0 \times 10^{-14}$ \\
$10 \mathrm{wt} \%$ Untreated & $51.5 \pm 1.1$ & $11.7 \pm 0.6$ & $2.4 \times 10^{-14}$ \\
$2 \mathrm{wt} \%$ C3-treated & $31.4 \pm 1.3$ & $3.5 \pm 0.7$ & $2.1 \times 10^{-14}$ \\
$5 \mathrm{wt} \%$ C3-treated & $48.3 \pm 1.5$ & $8.5 \pm 0.3$ & $3.1 \times 10^{-14}$ \\
$10 \mathrm{wt} \%$ C3-treated & $49.8 \pm 1.0$ & $13.2 \pm 0.1$ & $3.0 \times 10^{-14}$ \\
\hline
\end{tabular}

